\title{
Kernos
}

Revue internationale et pluridisciplinaire de religion grecque antique

16 | 2003

Varia

\section{P. SCARPI (éd.), Le Religioni dei misteri. Vol. I \& II}

\section{André Motte}

\section{(2) OpenEdition}

\section{Journals}

Édition électronique

URL : http://journals.openedition.org/kernos/841

DOI : 10.4000/kernos.841

ISSN : 2034-7871

Éditeur

Centre international d'étude de la religion grecque antique

Édition imprimée

Date de publication : 1 janvier 2003

Pagination : 365-366

ISSN : 0776-3824

Référence électronique

André Motte, «P. scarpI (éd.), Le Religioni dei misteri. Vol. I \& II », Kernos [En ligne], 16 | 2003, mis en ligne le 14 avril 2011, consulté le 23 septembre 2020. URL : http://journals.openedition.org/kernos/ 841 ; DOI : https://doi.org/10.4000/kernos.841 
Dans la mesure où, par son refus, Achille a menacé la survie de la communauté, la vraie conclusion de l'Iliade est moins à attendre de la réconciliation avec Agamemnon, qui intervient dès le chant XIX, que de la confrontation du héros avec un père, non pas le sien, mais celui d'Hector, venu réclamer la dépouille de son fils, au chant XXIV. Ramené à la raison des ancêtres par Priam, Achille réintègre sa place dans l'ordre des générations (Conclusion, p. 415-436). Le poème apparaît donc comme un garant de la cohésion sociale par l'inscription de l'individu dans la tradition de ses pères.

L'ouvrage a été soutenu comme thèse de doctorat en 1998 à l'Université de Genève. L'exposé très systématique, accompagné d'une importante bibliographie et d'index, est parfois ralenti par des répétitions ou par des citations redondantes, et ce que l'analyse gagne en rigueur, elle le perd quelque peu en lisibilité. Mais on appréciera de voir se développer, au fil d'une lecture érudite des poèmes, la vision d'un interprète minutieux, qui n'a pas reculé devant l'audace de se mesurer à une ouvre comme l'Iliade. Même si les arguments semblent quelques fois ténus, la thèse avancée ici est séduisante et jette indubitablement un éclairage neuf sur une œuvre si connue et pourtant toujours à découvrir.

O. Gengler (EHESS-Paris)

Paolo Scarpi (éd.), Le Religioni dei misteri. Vol. I: Eleusi, Dionisismo, Orfismo. Vol. II: Samotracia, Andania, Iside, Cibele e Attis, Mitraismo, Milano, Arnaldo Mondadori Editore, Fondazione Lorenzo Valla, 2002. 2 vol. $13 \times 20 \mathrm{~cm}$, XLVII $+616 \mathrm{p}$. \& LXXXII + 692 p. (Scrittori greci e latini). ISBN : 88-04-50667-9 \& 88-04-50317-3.

Voici un volumineux et important ouvrage dont il convient d'annoncer sans délais la parution, quitte à se borner ici à une présentation essentiellement descriptive. Il s'agit, en effet, d'un recueil des témoignages écrits, littéraires et épigraphiques (à l'exclusion donc des documents archéologiques et iconographiques) relatifs aux principaux cultes à mystères de l'Antiquité gréco-romaine, et ce recueil se signale par une double originalité. D'une part, à considérer en particulier chacun des huit cultes visés, c'est la première fois que sont en même temps rassemblés méthodiquement, traduits et commentés les textes anciens qui nous les font connaître. D'autre part, la réunion de ces textes dans un même ouvrage permet une vue d'ensemble des mystères antiques et incite d'autant plus à une démarche comparative que les témoignages sont, pour chaque culte, regroupés sous les mêmes rubriques, si ce n'est que les plus richement documentés peuvent requérir une ou deux rubriques supplémentaires (e.g., "Petits et grands mystères » pour Éleusis), tandis que d'autres affichent quelquefois une lacune. Le tronc commun est le suivant: « Mythes de fondation ", «Sacerdoce », "Culte et pratiques rituelles », «Initiation », "Secret et interdictions ", "Eschatologie » ou "Sotériologie »; la numérotation des témoignages est faite par rubrique, chacune de celles-ci étant précédée d'une lettre. Eu égard à ce qui caractérise les cultes à mystères, cette division semble pertinente et permet à tout le moins une mise en ordre fort bienvenue des témoignages; le cas particulier des textes relevant de deux ou de plusieurs rubriques, - songeons par exemple à l'Hymne bomérique à Déméter, cité ici in extenso -, est résolu par des reproductions partielles ou par des renvois.

Dans une substantielle introduction de quarante pages, l'A. s'attache tout d'abord à éclairer la notion de mystères, en référence notamment à la terminologie dont usent

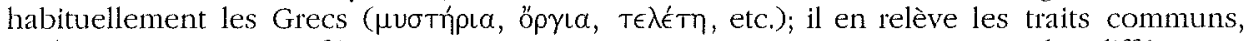
souligne certaines interférences et connexions, mais insiste tout autant sur les différences, récusant à cet égard la tendance à une homogénéisation qu'on observe déjà dans l'Antiquité tardive. Est aussi abordée, avec beaucoup de réserve, la question difficile des origines. L'A. indique encore deux limites données à son travail et s'en explique. Il est évident tout d'abord que le nombre de "mystères " qu'évoquent les sources anciennes en usant du vocabulaire susdit est bien supérieur à huit; il suffit, pour s'en convaincre, de suivre Pausanias dans ses pérégrinations à travers le Péloponèse, par exemple. Les cultes omis dans le présent ouvrage, explique l'A., l'ont été soit en raison de la pauvreté de l'information qui les concerne, soit parce qu'il apparaît, à l'examen, que les connotations mystériques que leur prêtent certains Anciens trop prompts aux assimilations ne se justifient pas; tel est le cas, par exemple, des cultes d'Adonis. Dresser ici ne serait-ce qu'un 
simple inventaire des cultes non retenus n'eût pas été inutile. La seconde limitation est plus importante et devia rester présente à l'esprit quand on utilisera l'ouvrage. C'est qu'une sélection a été également opérée parmi les témoignages relatifs aux huit complexes mythico-rituels traités; l'A. parle d'ailleurs lui-même d'« anthologie ». Ont été négligés, précise-t-il, les témoignages obscurs que la science philologique et l'épigraphie n'ont pas réussi à tirer au clair. Seul l'usage, joint à un examen approfondi, permettra de juger de la pertinence des choix ici opérés. Précisons encore que les textes originaux ne sont pas accompagnés d'un apparat critique. Pour comprendre ces options, il convient de noter que l'A. présente lui-même son ouvre comme celle d'un historien des religions plutôt que celle d'un philologue de métier. Signalons enfin qu'une introduction beaucoup plus brève inaugure aussi le second volume; on y esquisse notamment les contextes historiques nouveaux dans lesquels se cléveloppent les cultes mystériques durant les périodes hellénistique et romaine. On aura compris que le premier volume regroupe quant à lui les trois cultes les plus anciens, ou du moins les plus anciennement attestés. On observera toutefois que les mystères de Samothrace, qui inaugurent le second volume, sont déja mentionnés par Hérodote; mais c'est là, à vrai dire, l'unique témoignage littéraire un peu circonstancié qui remonte à l'époque classique.

Tant s'en faut que les huit cultes retenus (le mot « culte » parait plus approprié, en l'occurrence, que celui de "religions ") aient connu, dans l'Antiquité, le même rayonnement et suscité la même curiosité chez les lettrés. Aussi bien le nombre de témoignages qui les concernent varie-t-il considérablement, de même que l'ampleur des commentaires qui leur sont consacrés par l'A. Les mystères d'Éleusis, on s'y attendait, occupent à eux seuls plus du quart de l'ouvrage (219 témoignages au total, 120 pages de commentaires), les cultes les moins bien documentés étant ceux d'Andanie (18 témoignages, 13 pages de commentaires). Entre ces deux extrêmes, mais à bonne distance du premier, les mystères dionysiaques, les mystères de Samothrace et les mystères orphiques (comprenant notamment les fameuses «lamelles ») sont, clans l'ordre, les mieux représentés quant au nombre de témoignages, mais ce sont les cultes mystériques de Dionysos et ceux d'Isis, - c'est sur ces derniers qu'a porté la collaboration de B. Rossignoli, - qui suscitent les commentaires les plus abondants. Précédant chacune des huit anthologies, une brève introduction permet de situer historiquement les cultes concernés et de les caractériser sommairement.

Une bibliographie générale, forte de quelque cent soixante titres et reproduite dans les deux volumes, recense de façon sélective les études d'ensemble qui concernent les mystères et les religions du monde antique, outre les divers répertoires de textes et de fragments utilisés; la plupart des publications retenues ont vu le jour dans les trente dernières années, mais les "classiques » plus anciens ne sont pas négligés. Viennent ensuite, réparties entre les deux volumes, les bibliographies spéciales, pareillement sélectives, qui se rapportent aux huit cultes étudiés.

La présentation de l'ouvrage est soignée et sa consultation commode. Les pages où sont reproduits les textes originaux et les traductions qui leur font face sont bien aérées. Annoncés par le signe « < en marge des traductions et rejetés à la fin des deux volumes, les commentaires ponctuels suivent l'ordre des témoignages et reprennent leur numérotation. La table des matières qui clôture chaque volume énumère entre autres tous les témoignages produits en reprenant les rubriques, les numéros d'ordre des textes ainsi que leurs références, ce qui donne d'emblée un aperçu des sources propres à chacun des cultes et permet d'y discemer aisément d'éventuelles lacunes. Un index des sources anciennes, général cette fois, figure aussi à la fin du second volume, précédé d'un index des noms de divinités et de héros, et suivi d'un index des éditions et des traductions de textes utilisées.

On le voit, l'A. n'a pas ménagé sa peine pour rendre facilement accessible au lecteur la documentation très riche qu'il met à sa disposition. Nul doute que cette précieuse somme ne rende de grands services aux chercheurs et ne contribue ainsi à élargir et à affiner notre connaissance des mystères antiques. 